\title{
Scaled variational computation of the energy spectrum of a two-dimensional hydrogenic donor in a magnetic field of arbitrary strength
}

\author{
Ramiro Pino* and Víctor M. Villalba ${ }^{\dagger}$ \\ Centro de Física, \\ Instituto Venezolano de Investigaciones Científicas, IVIC \\ Apdo 21827, Caracas 1020-A, Venezuela
}

February 28, 2018

\begin{abstract}
We compute the energy levels of a 2D Hydrogen atom when a constant magnetic field is applied. With the help of a mixed-basis variational method and a generalization of virial theorem, which consists in scaling the wave function, we calculate the binding energies of the $1 \mathrm{~S}, 2 P^{-}$and $3 D^{-}$levels. We compare the computed energy spectra with those obtained via a generalization of the mesh point technique as well as the shifted $1 / N$ method. We show that the variational solutions present a very good behavior in the weak and strong magnetic field regimes.
\end{abstract}

In the past few years a great effort has been devoted to the study of two-dimensional atoms. The technological advances in semiconductor processing has enabled to manufacture nanostructures where the electrons are confined to two dimensional (quantum wells), one dimensional (quantum wires) and zero dimensional (quantum dots) mo-

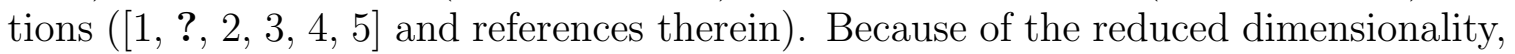
new and interesting transport and optical properties can be observed. In order to explain the experimental data, a large number of theoretical investigations have been performed [6, 7, 8].

Probably the best known quantum well configuration consists of regions of GaAs which act as wells of conduction electrons, separated by regions of $\mathrm{Ga}_{1-x} \mathrm{Al}_{x} \mathrm{As}$ which act as barriers. The application of magnetic field perpendicular to the quantum wells is expected to provide further band-structure data and binding energy information.

*e-mail: rpino@ivic.ivic.ve

†e-mail: villalba@ivic.ivic.ve 
The importance of the theoretical calculations demands reliable analytical and numerical techniques. In this study we are motivated to analyze the binding energies of the ground and excited states of a hydrogenic donor in a 2D electron gas. Here, with the help of a scaled mixed variational method, we compute the energy levels of a $2 \mathrm{D}$ Hydrogenic atom in the presence of a magnetic field of arbitrary strength,we compare our results with those obtained by numerical computation and with the shifted $1 / N$ method [9, 10, 11].

The Hamiltonian describing the electromagnetic interaction between a conduction electron and a donor impurity center when a constant magnetic field $\mathrm{B}$ is applied perpendicular to the $\mathrm{x}-\mathrm{y}$ plane can be written as

$$
H=-\nabla^{2}+\gamma L_{z}-\frac{2}{\rho}+\frac{\gamma^{2} \rho^{2}}{4}
$$

where we have written the vector potential $\mathbf{A}=\frac{B}{2}(-y, x, 0)=\frac{B r}{2} \hat{e}_{\varphi}$ in the symmetric gauge. The coupling constant $\gamma$, which measures the ratio between the magnetic energy and Coulomb energy, is defined as $\gamma=\epsilon^{2} \hbar^{3} B /\left(c e^{3} m^{* 2}\right)$ where $m^{*}$ is the effective mass, $\epsilon$ the dielectric constant of the host material, $\nabla^{2}$ is the two-dimensional Laplacian, and $L_{z}$ is the angular momentum operator $-i \hbar \partial / \partial \phi$ with eigenvalue $\hbar m$. The units of energy are given in terms of the effective Rydberg constant $\mathcal{R}_{0}^{*}=m^{*} e^{4} / 2 \hbar^{2} \epsilon^{2}$ and the effective Bohr radius $a^{*}=\hbar^{2} \epsilon / m^{*} e^{2}$, respectively.

The substitution

$$
\Psi(\mathbf{r})=e^{i m \varphi} \Psi(\rho),
$$

reduces the Schrödinger equation $H \Psi=E \Psi$ to the following second order ordinary differential equation

$$
\left[-\frac{d^{2}}{d \rho^{2}}-\frac{1}{\rho} \frac{d}{d \rho}+\frac{m^{2}}{\rho^{2}}+\frac{\gamma^{2} \rho^{2}}{4}-\frac{2}{\rho}+m \gamma-E\right] \Psi(\rho)=0 .
$$

Exact solutions of eq. (3) cannot be expressed in closed form in terms of special functions. There are analytical expressions for the energy for particular values of $\gamma$ and $m$ [12, 13, 14]. Besides numerical and perturbation methods [15, 16], different techniques have been used in order to obtain the eigenvalues $E$ in equation (3), in particular the two-point Padé approximation [17, 18]

Recently, using the shifted 1/N expansion, Mustafa [11, 19] has computed the $1 S$, $2 P^{-}$and $3 D^{-}$energy levels for a $2 \mathrm{D}$ donor impurity in the presence of a magnetic field. The shifted $1 / N$ and Padé methods are very powerful in computing the energy spectra but fail in giving a reasonable simple wave function and general criteria for the lower bound of energy values. In the present article we discuss the problem using a scaled two-terms mixed-basis variational approach. We compare our results with those obtained using the Schwartz [20] interpolation technique, as well as the shifted $1 / N$ method; showing that our results fit very well to those computed numerically for any range of values of the magnetic strength $B$. 
In order to apply the variational method to our problem [21, we look for a trial wave function. Since equation (3) reduces to the Hydrogen atom equation when $\gamma=0$, we can consider as a basis for $\gamma<<1$ the Hydrogen wave functions $\Psi_{H}$. Since $<\Psi_{H}|H| \Psi_{H}>\geq E$, we obtain a lower bound of the energy for small values of the parameter $\gamma$. The solution of equation (3) when $\gamma=0$ is

$$
\Psi_{H}(\rho)=D_{m, n} e^{-\rho /\left(1 / 2+n_{\rho}+|m|\right)} \rho^{|m|} L\left(n_{\rho}, 2|m|, \frac{2 \rho}{\left(1 / 2+n_{\rho}+|m|\right)}\right)
$$

where $D_{m, n}$ is a normalization constant, and L $(\mathrm{a}, \mathrm{b}, \mathrm{x})$ are the Laguerre polynomials 223. Consequently the energy spectrum in the zero-field limit takes the form

$$
E_{H}=-\frac{1}{\left(1 / 2+n_{\rho}+|m|\right)^{2}}
$$

Conversely, for large values of $\gamma$, a good trial basis is that of the spherical oscillator. In this case the solution of equation (3) has the form

$$
\Psi_{O s c}(r)=C_{m, n} e^{-\gamma \rho^{2} / 4} \rho^{|m|} L\left(n_{\rho},|m|, \frac{\gamma}{2} \rho^{2}\right)
$$

and in the high-field limit, the energy levels are

$$
E_{O s c}=\gamma\left(2 n_{\rho}+|m|+m+1\right) .
$$

If we attempt to apply the variational method using the hydrogen atom basis, we will obtain good agreement with the accurate results for small values of $\gamma$, but this approach fails for large $\gamma$ even if we consider a many term basis. An analogous situation occurs when we use the oscillator basis, in which case we obtain a good agreement for large $\gamma$ but the convergence is very slow for small values of $\gamma$ [22]. In order to solve this problem, we propose a mixed-basis approach. The idea is to use as trial function, for any quantum level, a linear combination of the form

$$
\Psi=c_{H} \Psi_{H}+c_{O} \Psi_{O s c}
$$

where $\Psi_{H}$ and $\Psi_{O s c}$ are the corresponding hydrogen and oscillator wave functions associated with the quantum level in question; $c_{O}$ and $c_{H}$ are constants to be calculated. It is worth noticing that our mixed basis is not orthogonal under the inner product $\int_{0}^{\infty} \Psi_{i} \Psi_{j} \rho d \rho$. We proceed to minimize the expectation value $\langle\Psi|H| \Psi\rangle$ with the normalization condition, $\langle\Psi \mid \Psi\rangle=1$

Applying the variational approach to the basis coefficients $c_{i}$, we reduce our problem to that of solving the matrix equation

$$
\left[\left\langle\Psi_{i}|H| \Psi_{j}\right\rangle-\lambda\left\langle\Psi_{i} \mid \Psi_{j}\right\rangle\right] c_{j}=0
$$


whence after substituting the Hamiltonian (11) into (9), and taking into account the separated differential equation (3) we obtain the matrix equation,

$$
\left(\begin{array}{ll}
E_{H}+\frac{\gamma^{2}}{4} A+m \gamma-\lambda & \left(E_{O s c}-\lambda\right) S-2 C \\
\left(E_{O s c}-\lambda\right) S-2 C & E_{O}-2 B-\lambda
\end{array}\right)\left(\begin{array}{l}
c_{1} \\
c_{2}
\end{array}\right)=\left(\begin{array}{l}
0 \\
0
\end{array}\right)
$$

where

$$
\begin{array}{r}
\left\langle\Psi_{H}\left|\rho^{2}\right| \Psi_{H}\right\rangle=A,\left\langle\Psi_{O s c}\left|\rho^{2}\right| \Psi_{H}\right\rangle=D,\left\langle\Psi_{O s c}\left|\frac{1}{\rho}\right| \Psi_{H}\right\rangle=C, \\
\left\langle\Psi_{O s c}\left|\frac{1}{\rho}\right| \Psi_{O s c}\right\rangle=B,\left\langle\Psi_{H} \mid \Psi_{0}\right\rangle=S
\end{array}
$$

where the lowest value of $\lambda$ will be the energy of the level. Since we obtain a second order equation for $\lambda$, we can analytically compute the energy eigenvalues and eigenvectors. The advantage of this approach is twofold. First, because of variational approximation, the eigenvalues satisfy the inequality $\lambda \geq E$ and therefore we have a lower bound for our energy levels. Second, we obtain a relatively simple expression for the normalized eigenfunctions.

Instead of restricting ourselves to the the energy values obtained from (9) via the mixed-basis variational method, we can improve the accuracy of the energy with the help of the virial theorem. Using this technique, after re-scaling the radial parameter $r \rightarrow \xi r$ we have that

$$
\Psi(\rho) \rightarrow \Psi(\xi \rho)
$$

and the matrix terms associated with $\rho^{n}$ and the second derivative become

$$
\begin{gathered}
\left\langle\Psi_{i}\left|\rho^{n}\right| \Psi_{j}\right\rangle \rightarrow \xi^{-n}\left\langle\Psi_{i}(\xi r)\left|(\xi \rho)^{n}\right| \Psi_{j}(\xi \rho)\right\rangle=\xi^{-n}\left\langle\Psi_{i}\left|\rho^{n}\right| \Psi_{j}\right\rangle \\
\left\langle\Psi_{i}\left|\frac{d}{d \rho^{2}}\right| \Psi_{j}\right\rangle \rightarrow \xi^{2}\left\langle\Psi_{i}(\xi \rho)\left|\frac{d}{d(\xi \rho)^{2}}\right| \Psi_{j}(\xi \rho)\right\rangle=\xi^{2}\left\langle\Psi_{i}\left|\frac{d}{d \rho^{2}}\right| \Psi_{j}\right\rangle
\end{gathered}
$$

Then, with the help of the virial theorem for a Coulomb and a radial oscillator potential,

$$
\begin{gathered}
E_{H}=-\left\langle\Psi_{H}\left|T_{H}\right| \Psi_{H}\right\rangle=\frac{1}{2}\left\langle\Psi_{H}\left|V_{H}\right| \Psi_{H}\right\rangle \\
E_{O s c}=2\left\langle\Psi_{O s c}\left|T_{O s c}\right| \Psi_{O s c}\right\rangle=2\left\langle\Psi_{O s c}\left|V_{O s c}\right| \Psi_{O s c}\right\rangle
\end{gathered}
$$

where $T$ is the kinetic energy term, the re-scaled Hamiltonian reduces to

$$
\begin{gathered}
H=\left(\begin{array}{cc}
H_{11}(\xi) & H_{12}(\xi) \\
H_{21}(\xi) & H_{22}(\xi)
\end{array}\right)= \\
\left(\begin{array}{ll}
\left(\xi(2-\xi) E_{H}+\frac{\gamma^{2}}{4 \xi^{2}} A+m \gamma\right. & \xi^{2} J+\frac{\gamma^{2}}{4 \xi^{2}} D-2 \xi C+m S \\
\xi^{2} J+\frac{\gamma^{2}}{4 \xi^{2}} D-2 \xi C+m S & \frac{1}{2}\left(\xi^{2}-\xi^{-2}\right)\left(E_{O s c}-m \gamma\right)-2 \xi B+m \gamma
\end{array}\right)
\end{gathered}
$$


with

$$
J=\int_{0}^{\infty}\left(\frac{d \Psi_{H}}{d \rho} \frac{d \Psi_{O s c}}{d \rho}+m^{2} \Psi_{H} \Psi_{O s c}\right) \rho d \rho .
$$

In order to compute the quantum energy values, we proceed to compute the lowest eigenvalue of the matrix equation given by

$$
\left(\begin{array}{ll}
H_{11}(\xi)-\lambda & H_{12}(\xi)-\lambda S \\
H_{12}(\xi)-\lambda S & H_{22}(\xi)-\lambda
\end{array}\right)\left(\begin{array}{l}
c_{1} \\
c_{2}
\end{array}\right)=\left(\begin{array}{l}
0 \\
0
\end{array}\right)
$$

which takes the form

$$
\begin{array}{r}
\lambda=\frac{H_{11}+H_{22}-2 S H_{12}}{2\left(1-S^{2}\right)} \\
-\frac{\sqrt{\left(H_{11}+H_{22}-2 S H_{12}\right)^{2}-4\left(1-S^{2}\right)\left(H_{11} H_{22}-H_{12}^{2}\right)}}{2\left(1-S^{2}\right)} .
\end{array}
$$

Since the components of $H$ depend on the scale parameter $\xi, \lambda$ is a function of $\xi$, and the optimal value for $\xi$ can be obtained computing the minimum for $\lambda(\xi)$, which reduces to solving the algebraic equation

$$
\frac{d \lambda}{d \xi}=0
$$

Then, we can substitute the value of $\xi$, obtained after solving (20), into (11) and obtain an analytic expression for the scaled wave functions. As illustration of the mixed-basis method, we present two different expansions of the ground state of th 2D Hydrogen Hamiltonian (11). We plot the energy against $\gamma^{\prime}=\gamma /(\gamma+1)$ as the horizontal scale.

For comparison, the numerical computations of the energy spectra associated with equation (3) are carried out with the help of the Schwartz method 20] which is a generalization of the mesh point technique for numerical approximation of functions. This method gives highly accurate results given a thoughtful choice of the reference function. For equation (3) we chose as the interpolation function

$$
f(\rho)=\sum_{m} f_{m} \frac{u(\rho)}{\left(\rho-r_{m}\right) a_{m}}
$$

where

$$
u(\rho)=\sin \left[\pi(\rho / h)^{1 / 2}\right] .
$$

Here $r_{m}$ is a zero of $u(\rho), a_{m}$ is a zero of its derivative, and $h$ is the step of the quadratic mesh. Here, as illustration of the mixed-basis method, we present two different expansions of the ground state of the 2D Hydrogen Hamiltonian

Figures 1, 3, and 5 compare the variational results with those obtained numerically and with the help of the shifted $1 / N$ method. 


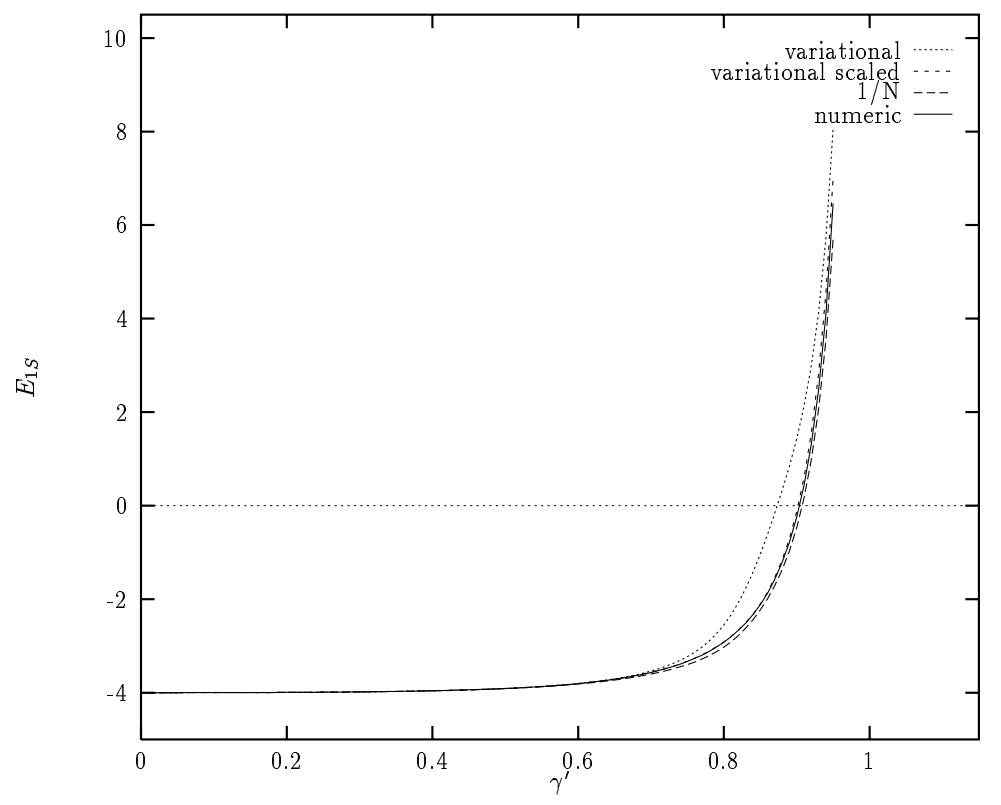

Figure 1: Energy of the $1 S$ state as a function of $\gamma^{\prime}$. The thin solid line is obtained by numerical methods; the dotted line is obtained using the mixed $1 \mathrm{~S}$ hydrogen and $1 \mathrm{~S}$ oscillator bases variational method. The thin broken line is obtained via the scaled variational method with the $1 \mathrm{~S}$ hydrogen and oscillator bases. The thick broken line is obtained with the help of the shifted $1 / N$ method 


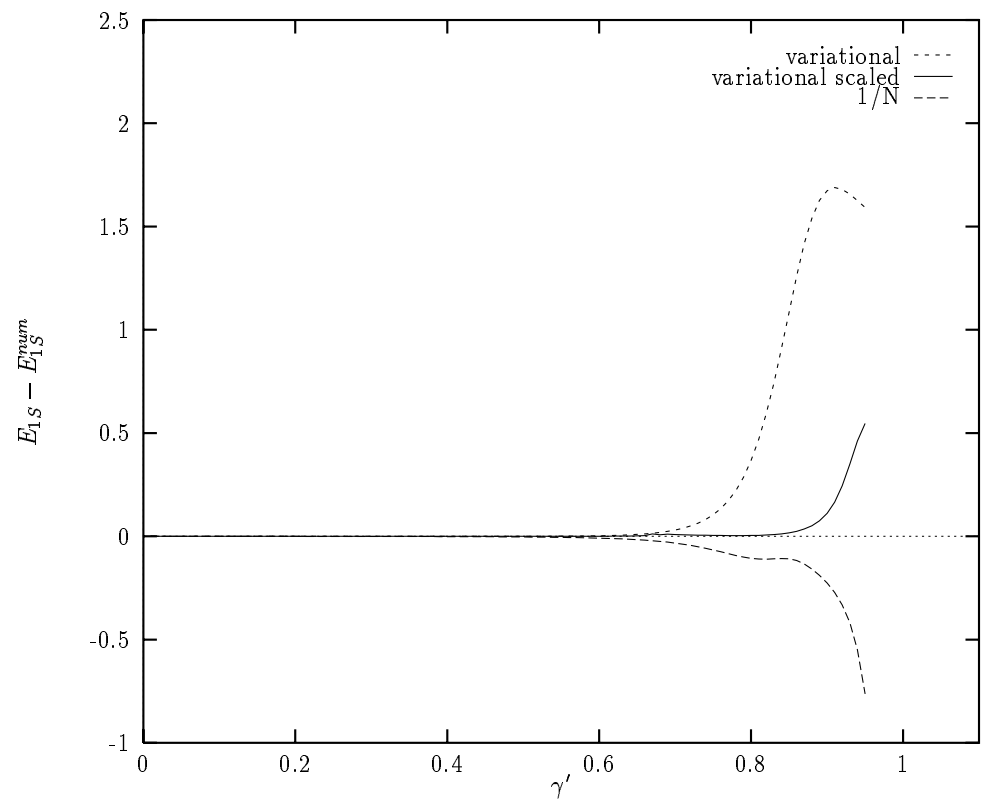

Figure 2: The figure shows the difference between the numerical result for the $1 S$ energy spectrum and the energy values computed with the help of the mixed variational (thin broken line), scaled variational (thin solid line), and the shifted $1 / N$ method (thick broken line) 


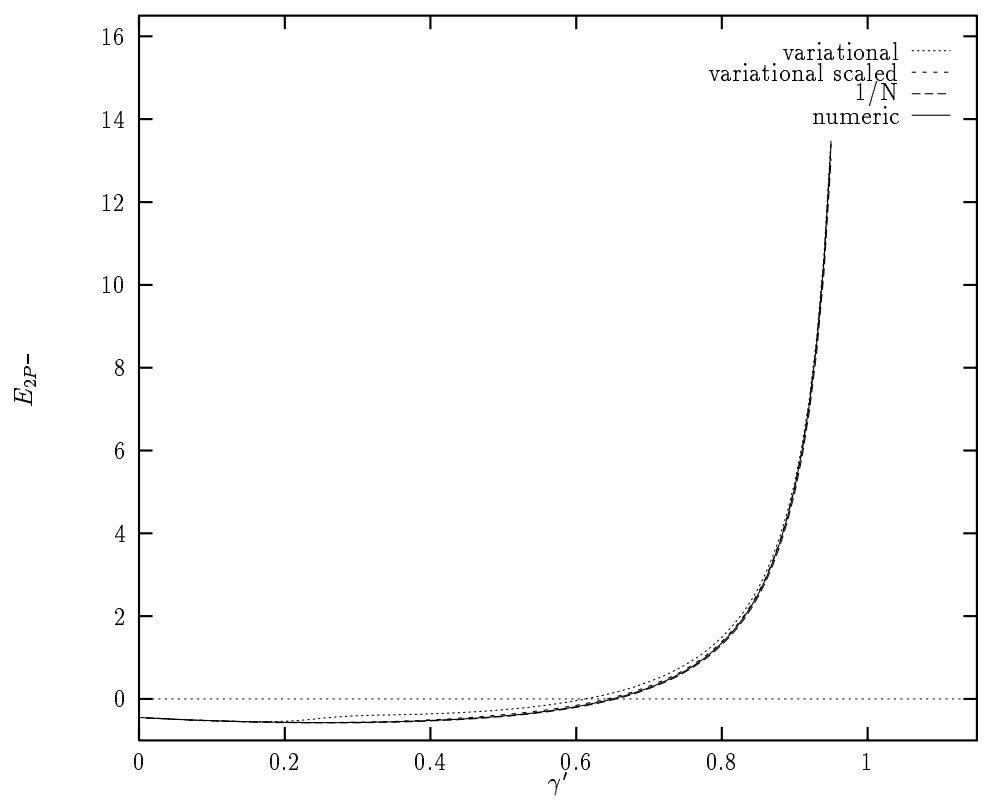

Figure 3: Energy of the $2 P^{-}$state as a function of $\gamma^{\prime}$. The thin solid line is obtained by numerical methods; the dotted line is obtained using the mixed $2 P^{-}$hydrogen and $2 P^{-}$oscillator bases variational method. The thin broken line is obtained via the scaled variational method with the $2 P^{-}$hydrogen and oscillator bases. The thick broken line is obtained with the help of the shifted $1 / N$ method 


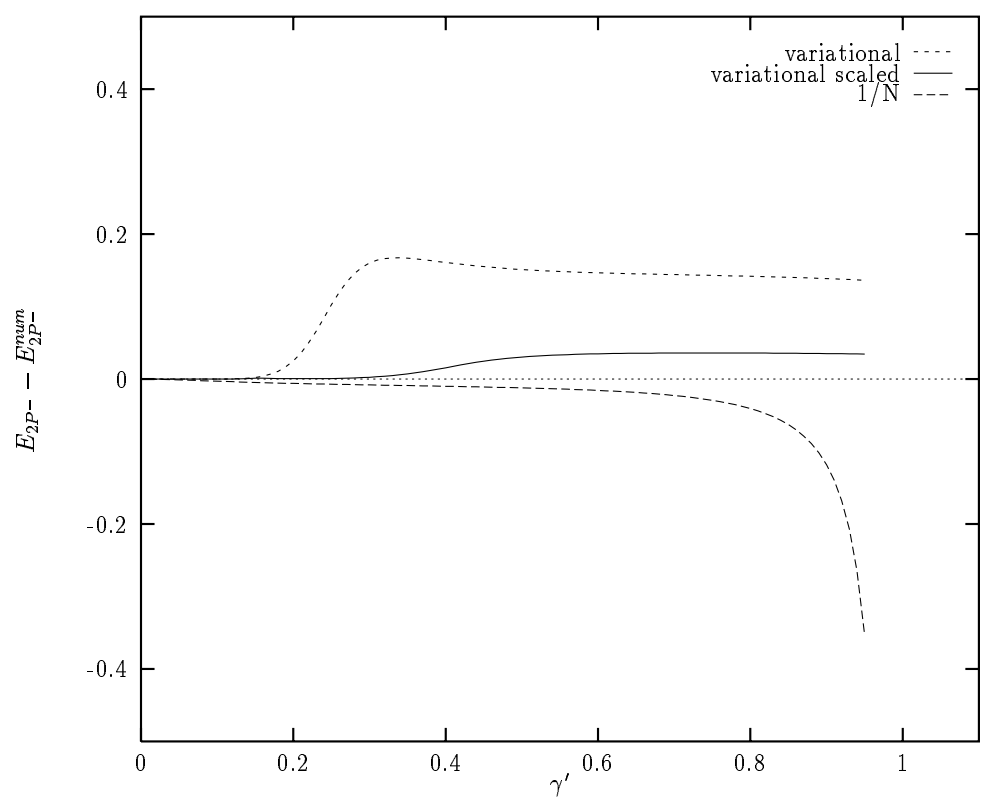

Figure 4: The figure shows the difference between the numerical result for the $2 P^{-}$ energy spectrum and the energy values computed with the help of the mixed variational (thin broken line), scaled variational (thin solid line), and the shifted $1 / N$ method (thick broken line) 


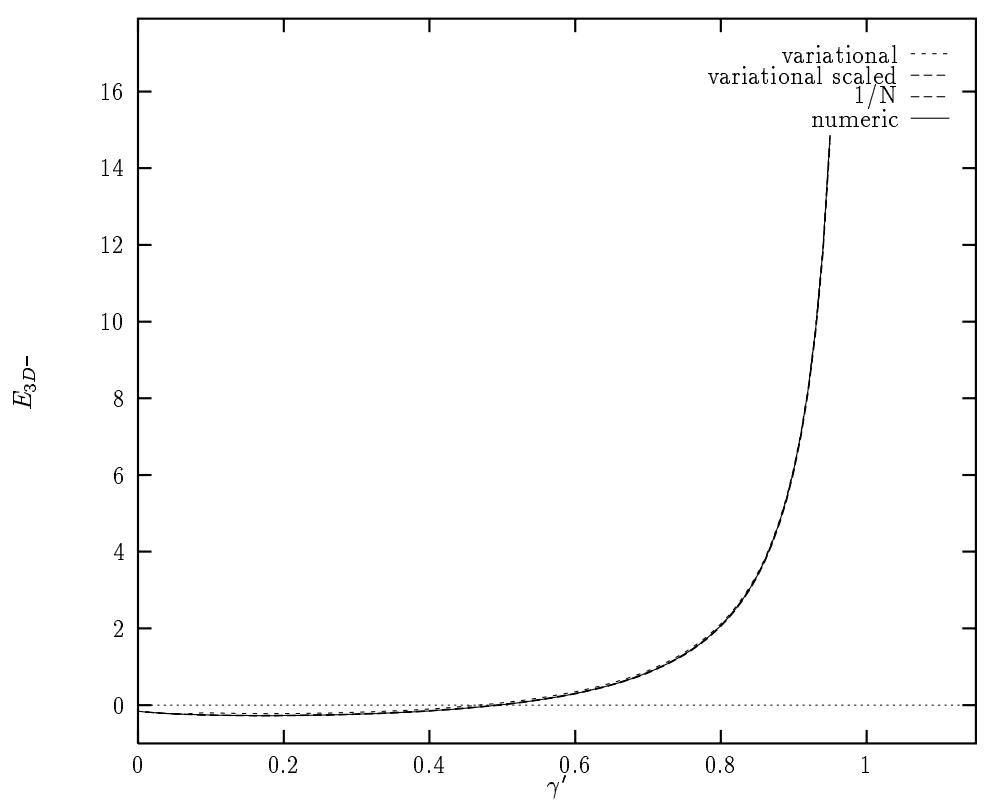

Figure 5: Energy of the $3 D^{-}$state as a function of $\gamma^{\prime}$. The thin solid line is obtained by numerical methods; the dotted line is obtained using the mixed $3 D^{-}$hydrogen and $3 D^{-}$oscillator bases variational method. The thin broken line is obtained via the scaled variational method with the $3 D^{-}$hydrogen and oscillator bases. The thick broken line is obtained with the help of the shifted $1 / N$ method 


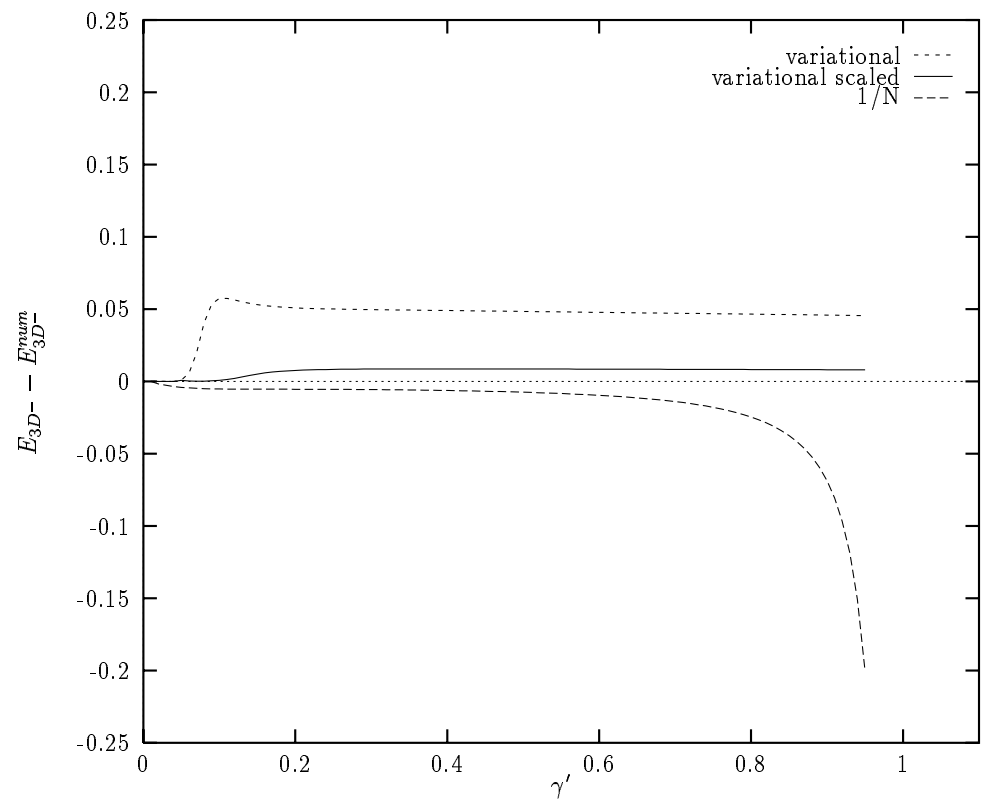

Figure 6: The figure shows the difference between the numeric result for the $3 D^{-}$energy spectrum and the energy values computed with the help of the mixed variational (thin broken line), scaled variational (thin solid line), and the shifted $1 / N$ method (thick broken line) 
It is easy to see that even for a two term mixed basis a good fitting is obtained in the weak and strong field regimes. One of the bases gives a reasonable good fitting in the intermediate region. A better fit is obtained with the help of the scaled variational method. We also have that for the $2 P^{-}$and $3 D^{-}$states the mixed-basis variational approach gives very good results.

Examining Figs. 1, 3 and 5, it is not obvious which technique gives the most accurate results for computing the hydrogen energy levels. However, Figures 2, 4 and 6 show that the shifted $1 / N$ method always gives results below the the numerical energy levels. Among the three analytic methods, figures 2, 4 and 6 show that the scaled variational method gives the most close to numerical results even for large values of $\gamma$.

It would be interesting to apply the mixed-basis technique as well as the scaled variational method for the $2 \mathrm{D}$ Hydrogen problem when relativistic effects are not negligible. This will be the object of a forthcoming publication.

\section{Acknowledgments}

We thank Dr. Juan Rivero for helpful discussions. This work was supported by CONICIT under project 96000061 .

\section{References}

[1] G. BASTARD Phys. Rev. B., 24 4714. (1981)

[2] R. L. GREENE, and K. K. BAJAJ Solid State Communications, 45, 831. (1983)

[3] R. L. GREENE, and K. K. BAJAJ Phys. Rev. B., 31, 913. (1985).

[4] C. SIKORSKI and U. MERKT Phys. Rev. Lett. 62, 2164. (1989)

[5] M. A. KASTNER Rev. Mod. Phys., 64, 1849. (1992)

[6] P. A. MAKSYM and T. CHAKRABORTY Phys. Rev. Lett., 65, 108. (1990)

[7] N. F. JOHNSON and M. C. PAYNE Phys. Rev. Lett., 67, 1157. (1991)

[8] C. R. PROETTO Phys. Rev. Lett., 76, 2824. (1996)

[9] T. IMBO, A. PAGNAMENTA and U. SUKHATME Phys. Rev B., 31, 913 (1984)

[10] T. IMBO, and U. SUKHATME Phys. Rev. D., 31, 2655. (1985)

[11] O. MUSTAFA J. Phys: Condensed Matter 5, 1327. (1993) 
[12] E. D. LOZANSKII and O. B. FIRSOV Izv. Vyssh. Ucheb. Zav. Fizika., 6, 52. (1974)

[13] M. TAUT J. Phys. A., 28, 2081. (1995)

[14] M. TAUT Phys. Rev A. 48, 3561 (1993)

[15] D. M. WHITTAKER and R. J. ELLIOT Solid State Commun., 68 , 1. (1988)

[16] G. DUGGAN. Phys. Rev. B., 37, 2759. (1988)

[17] A. H. MACDONALD and D. S. RITCHIE Phys. Rev B. 33, 8336. (1986)

[18] P. MARTin, J. J. RODRIGUEZ, and J. L. MARQUEZ Phys. Rev B., 45, 8359. (1992)

[19] O. MUSTAFA J. Phys. Condensed Matter., 8, 8673 (1996)

[20] C. SCHWARTZ J. Math. Phys., 26, 411. (1985)

[21] A. S. DAVYDOV.,Quantum Mechanics, Pergamon, Oxford, 1965.

[22] V. M. VILLALBA and R. PINO J. Phys: Condensed Matter. 8, 8667. (1996)

[23] N. N. LEBEDEV., Special functions and their applications, Dover, New York, 1972. 